\title{
Impaired osteoblastic differentiation, reduced bone formation, and severe osteoporosis in noggin-overexpressing mice
}

\author{
Xue-Bin Wu, ${ }^{1}$ Yanan $\mathrm{Li},{ }^{2}$ Adina Schneider, ${ }^{1}$ Wanqin Yu, ${ }^{1}$ Gopalan Rajendren, ${ }^{1}$ \\ Jameel Iqbal, ${ }^{1}$ Matsuo Yamamoto, ${ }^{3}$ Mohammad Alam, ${ }^{3}$ Lisa J. Brunet, ${ }^{4}$ Harry C. Blair, ${ }^{2}$ \\ Mone Zaidi, ${ }^{1}$ and Etsuko Abe ${ }^{1}$ \\ ${ }^{1}$ The Mount Sinai Bone Program, Mount Sinai School of Medicine, and the Bronx Veterans Administration Medical Center, \\ New York, New York, USA \\ ${ }^{2}$ Department of Pathology and Department of Cell Biology and Physiology, University of Pittsburgh School of Medicine, \\ and Veterans Administration Medical Center, Pittsburgh, Pennsylvania, USA \\ ${ }^{3}$ Division of Endocrinology and Metabolism and Center for Osteoporosis and Metabolic Bone Diseases, \\ University of Arkansas for Medical Sciences, Little Rock, Arkansas, USA \\ ${ }^{4}$ Division of Biochemistry and Molecular Biology, University of California, Berkeley, Berkeley, California, USA
}

\begin{abstract}
We describe the effects of the overexpression of noggin, a bone morphogenetic protein (BMP) inhibitor, on osteoblast differentiation and bone formation. Cells of the osteoblast and chondrocyte lineages, as well as bone marrow macrophages, showed intense $\beta$-gal histo- or cytostaining in adult noggin $^{+/-}$mice that had a LacZ transgene inserted at the site of noggin deletion. Despite identical BMP levels, however, osteoblasts of 20-month-old C57BL/6J and 4-month-old senescence-accelerated mice (SAM-P6 mice) had noggin expression levels that were approximately fourfold higher than those of 4-month-old C57BL/6J and SAM-R1 (control) mice, respectively. U-33 preosteoblastic cells overexpressing the noggin gene showed defective maturation and, in parallel, a decreased expression of Runx-2, bone sialoprotein, osteocalcin, and RANK-L. Noggin did not inhibit the ligandless signaling and prodifferentiation action of the constitutively activated BMP receptor type $1 \mathrm{~A}$, ca-ALK-3. Transgenic mice overexpressing noggin in mature osteocalcin-positive osteoblasts showed dramatic decreases in bone mineral density and bone formation rates with histological evidence of decreased trabecular bone and CFU-osteoblast colonies at 4 and 8 months. Together, the results provide compelling evidence that noggin, expressed in mature osteoblasts, inhibits osteoblast differentiation and bone formation. Thus, the overproduction of noggin during biological aging may result in impaired osteoblast formation and function and hence, net bone loss.
\end{abstract}

J. Clin. Invest. 112:924-934 (2003). doi:10.1172/JCI200315543.

\section{Introduction}

Bone morphogenetic protein-2 (BMP-2) and BMP-4 have established functions in embryonic skeletal development $(1,2)$. Both BMPs are expressed in the ventral region of the embryo and regulate mesodermal differentiation during gastrulation. Deletion of either the $B M P-2$ or $B M P-4$ gene therefore results in early embry-

Received for publication March 27, 2002, and accepted in revised form July 22, 2003.

Address correspondence to: Etsuko Abe, Division of Endocrinology, Diabetes, and Bone Diseases, Mount Sinai School of Medicine, One Gustave L. Levy Place, Box 1055, New York, New York 10029, USA. Phone: (212) 241-8735;

Fax: (212) 534-4820; E mail: etsuko.abe@mssm.edu.

Conflict of interest: The authors have declared that no conflict of interest exists.

Nonstandard abbreviations used: bone morphogenetic protein (BMP); UAMS-33 (U-33); alkaline phosphatase (ALP); nonspecific esterase (NSE); senescence-accelerated mice (SAM); macrophage colony-stimulating factor (M-CSF); tartrate-resistant acid phosphatase (TRAP); constitutively active ALK-3 (ca-ALK-3); hemagglutinin (HA); pegylated noggin (PEG-noggin); CFU fibroblast (CFU-F); CFU osteoblast (CFU-OB); osteocalcin gene 2 (OG-2); human growth hormone (hGH). onic lethality $(3,4)$. BMP-2 and BMP-4 have several other functions, including hair follicle growth, neuronal differentiation, cardiomyogenesis, thymocyte differentiation, and fusion of cranial sutures (5-9). All these effects are inhibited by the $60-\mathrm{kDa}$ homodimeric protein called noggin (10), as well as by other molecules, including chordin, gremlin, cerberus, and DAN (11). None of these inhibitors except noggin and chordin are expressed in bone cells of any lineage. Noggin binds with equal avidity to BMP- 2 and BMP-4 and competitively inhibits their interaction with the BMP receptor type $1 \mathrm{~A}$, ALK-3 $(12,13)$. In the embryo, noggin is expressed in the dorsal embryonic region where it regulates neural development (6); deletion of noggin thus results in postnatal mortality (14).

We recently observed that noggin was expressed in cells of the osteoblastic lineage (15). BMP-2, BMP-4, ALK-3, and noggin appeared during osteoblast differentiation with a defined time course, with the expression of BMP-4 preceding that of BMP-2 and noggin. Noggin potently inhibited the differentiation of osteoblasts in bone marrow cell cultures and preosteoblastic UAMS-33 (U-33) cells $(15,16)$. Similarly, 
reduced mineralization is noted in mice with intraperitoneally implanted noggin-containing matrigels (17). The application of BMP- 2 or an anti-noggin antibody reversed the effect of exogenous or endogenous noggin, respectively (15). Likewise, impaired osteoblast differentiation and bone loss in aged animals is partially curable by BMP-2 injection (18). Finally, noggin attenuated the formation of osteoclasts in both bone marrow cell cultures and stromal cell/hematopoietic cell cocultures (15). That this osteoclast-inhibitory action was mediated via osteoblasts was evident from our demonstration that the effect was abolished in the absence of stromal cell support. This effect was further consistent with the inhibition by noggin of the osteoblast-derived osteoclastogenic cytokine RANK-L (receptor activator for NF-אB ligand) (15). These studies suggested that noggin was not only a direct inhibitor of osteoblast differentiation but also an indirect regulator of osteoclast formation.

Here we investigate the role of noggin in osteoblast differentiation and adult skeletal remodeling. We evaluated the expression of noggin in adult mouse bone in vivo by examining $\beta$-gal staining and activity in heterozygous noggin ${ }^{+/-}$mice that had a LacZ gene inserted at the site of noggin gene deletion. Noggin was localized to osteoblasts, chondrocytes, and macrophages. Furthermore, BMP-2 regulated noggin expression in calvarial osteoblasts cultured ex vivo from these mice. Additionally, we found that infection of preosteoblastic U-33 cells with a retrovirus containing the noggin gene inhibited osteoblast differentiation and osteoclast-supporting activity. We generated transgenic mice that overexpress noggin in mature osteoblasts under the control of mouse osteocalcin promoter. The transgenic mice displayed dramatic osteoporosis, decreased trabecular and calvarial bone, diminished bone formation rates, and reduced osteoblast differentiation in ex vivo cultures. Finally, we showed that normally aged and SAM-P6 mice were functional noggin overexpressors. These studies provide strong evidence that the balance between the expression of BMP-2 (and BMP-4) and noggin may determine, in part, the extent of osteoblast differentiation, osteoclast formation, skeletal remodeling, and ultimately bone mass in adult mice.

\section{Methods}

Identification of $\beta$-gal-expressing cells in noggin ${ }^{+/-}$mice. Due to low BMP and noggin expression in adult animals, more sensitive methods than traditional in situ hybridization or immunohistostaining must be used. To detect noggin expression in situ, we used noggin ${ }^{+/}$ mice with the LacZ gene inserted at the site of noggin gene deletion. Note that noggin ${ }^{-/-}$mice die soon after birth due to a severe joint formation defect (14). The animal experiments were conducted following institutional Animal Care and Use Committee approval.

Tibia, spine, and calvaria from 4-month-old nog$\mathrm{gin}^{+/+}$and noggin ${ }^{+/-}$mice were embedded in OCT compound (Electron Microscopy Sciences, Washington,
Pennsylvania, USA), sectioned, and stained for $\beta$-gal by incubation with $1 \mathrm{mg} / \mathrm{ml} \mathrm{X-gal} \mathrm{solution} \mathrm{and}$ NP-40 overnight at $37^{\circ} \mathrm{C}(14)$. Noggin ${ }^{+/+}$mouse bone was used for negative control of $\beta$-gal staining. Calvarial osteoblasts were prepared by serial digestion of calvaria of 4-day-old noggin ${ }^{+/-}$mice with $200 \mathrm{U} / \mathrm{ml}$ collagenase type I. They were cultured at $2 \times 10^{4}$ in 48-well plates to examine the effect of various agents, including human recombinant BMP-2, -4 , and -6 , TGF- $\beta$, IGF-1, 1-34 human parathyroid hormone, noggin, and $1,25(\mathrm{OH})_{2} \mathrm{D}_{3}$ on expression of $\beta$-gal (noggin) and osteoblast differentiation. After 3 days of culture with factors and hormones, $\beta$-gal and alkaline phosphatase (ALP) activities were measured using the $\beta$-Galactosidase Enzyme Assay System (Promega Corp., Madison, Wisconsin, USA) and Diagnosis Kit 104 (Sigma-Aldrich, St. Louis, Missouri, USA), respectively. $\beta$-gal or ALP activity was expressed as units $/ \mathrm{min} / \mathrm{mg}$ protein or in nmol paranitrophenol $/ \mathrm{min} / \mathrm{mg}$ protein, respectively. Cellular $\beta$-gal activity in calvarial osteoblasts was detected by staining after incubation with substrate for 4-20 hours at $37^{\circ} \mathrm{C}$. Bone marrow cells prepared from 4-month-old noggin ${ }^{+/-}$mice were cultured for 1 week or 2 weeks in the presence of $1 \mathrm{mM}$ ascorbic acid-2phosphate (Wako Chemicals USA, Richmond, Virginia, USA) and stained for $\beta$-gal and then for ALP or nonspecific esterase (NSE) $(19,20)$.

Noggin expression in aged and senescence-accelerated mice. The expression of BMP-4 and noggin was measured in bone tissue (calvaria and long bones) and cultures of bone marrow cells of male 20-month-old (aged) C57BL/6J mice (National Institute on Aging, Bethesda, Maryland, USA) or 4-month-old senescence-accelerated mice (SAM-P6 mice; kindly provided by $\mathrm{T}$. Takeda, Kyoto University, Kyoto, Japan). The same tissues from 4-month-old (young) C57BL/6J mice or 4-month-old SAM-R1 control mice were analyzed in parallel for comparison.

For Western blotting, bone homogenates were lysed in RIPA buffer (10 mM phosphate buffer, $\mathrm{pH} 7.4,10 \%$ glycerol, $1 \%$ NP-40, 0.1\% SDS, 4 mM EDTA, $0.15 \mathrm{M}$ $\mathrm{NaCl}, 0.01 \mathrm{M} \mathrm{NaF}, 0.1 \%$ sodium orthovanadate, $1 \mathrm{mM}$ PMSF, $5 \mu \mathrm{g} / \mathrm{ml}$ trypsin inhibitor, and $5 \mu \mathrm{g} / \mathrm{ml}$ protease inhibitors), and cell lysates (100 $\mu$ g protein) were subjected to $10 \%$ SDS-PAGE (15). Noggin expression was detected using the anti-noggin antibody (provided by Regeneron Pharmaceuticals Inc., Tarrytown, New York, USA) and an horseradish peroxidase-goat anti-rat IgG. Band intensities for noggin expression were measured using a densitometer (Gel Doc documentation system; Bio-Rad Laboratories Inc., Hercules, California, USA) and expressed as a ratio after normalization to $\beta$-actin.

To quantify noggin and BMP-4 mRNA expression, fresh bone marrow cells from aged and SAM-P6 mice were cultured in the presence of $1 \mathrm{mM}$ ascorbic acid-2-phosphate for 4 weeks. Total RNA was prepared using the RNeasy mini kit (Qiagen Inc., Valencia, California, USA), and cDNA was generated using 
Superscript II (Invitrogen Corp., Carlsbad, California, USA). Expression of cDNA was quantitated by realtime PCR using specific primers and SYBR Green PCR Master Mix (Applied Biosystems, Foster City, California, USA). GAPDH was used to normalize BMP-4 and noggin expression. The data was expressed relative to the control group.

Osteoclast and macrophage preparation from bone marrow cells. Bone marrow cells were cultured for 2 days with 5 $\mathrm{ng} / \mathrm{ml}$ human macrophage CSF (M-CSF) and then nonadherent cells were purified using Ficoll-Paque Plus (Amersham Pharmacia Biotech Inc., Arlington Heights, Illinois, USA) as a source of hematopoietic osteoclast/macrophage progenitors (21). This cell culture fraction has minimal, if any, stromal/osteoblastic cell contamination. We checked for such contamination in parallel cultures by growing cells in the absence of $\mathrm{M}$-CSF, conditions under which macrophages are not viable while stromal cells continue to survive. We did not see any stromal cell growth in cultures without M-CSF, indicating a relatively high degree of purity of these Ficoll fractions. To enable osteoclast formation, the cells were subsequently incubated with $30 \mathrm{ng} / \mathrm{ml} \mathrm{M}$-CSF and $60 \mathrm{ng} / \mathrm{ml}$ glutathione S-transferase-RANK-L (provided by P.F. Ross at Washington University, St. Louis, Missouri, USA) for 5 days. Osteoclasts were stained with tartrate-resistant acid phosphatase (TRAP) using a kit (Sigma-Aldrich) per the manufacturer's instructions. Bone marrow macrophages were prepared from the progenitors by incubation with $30 \mathrm{ng} / \mathrm{ml}$ M-CSF for 3 days. Expression of BMP-4 and noggin mRNA and protein were measured using real-time PCR and Western blotting, respectively. The expression levels of noggin and BMP-4 were compared with those of U-33 preosteoblasts established from bone marrow cell cultures. Separate fractions of bone marrow macrophages were cultured in the presence or absence of $300 \mathrm{ng} / \mathrm{ml}$ BMP-2 with 30 $\mathrm{ng} / \mathrm{ml} \mathrm{M}$-CSF for 3 days to examine the regulation of noggin expression by Western blotting.

Noggin or constitutively active $A L K-3$ overexpression in preosteoblastic U-33 cells. Viral vector (pLXSN; Clontech Laboratories Inc., Palo Alto, California, USA) alone or vector containing either the murine noggin gene or the constitutively active $A L K-3$ (ca-ALK-3) gene were transfected into Phoenix cells (Phoenix cells kindly provided by G.P. Nolan, Stanford University, Palo Alto, California, USA) using Lipofectamine PLUS (Invitrogen Corp.) per the manufacturer's instructions. Two days after transfection, the cell supernatant containing virus particles was added to U-33 cell cultures (16) in the presence of $4 \mu \mathrm{g} / \mathrm{ml}$ Polybrene (Sigma-Aldrich). The empty vector $\mathrm{pLXSN}$ was used as control. Preliminary experiments using pLXSN-LacZ confirmed $100 \%$ infection of $U-33$ cells revealed by $\beta$-gal staining. The ca-ALK-3 plasmid (kindly provided by X. Cao, University of Alabama, Birmingham, Alabama, USA) has an HA influenza hemagglutinin (HA) tag at the C-terminus for protein detection, and a Glu ${ }^{233} \rightarrow$ Asp mutation in the $A L K-3$ gene, rendering it constitutively active and therefore allowing BMP signaling in the absence of BMP-2 or BMP-4 (22). Western blotting and immunocytostaining using anti-noggin and anti-HA antibodies confirmed the expression of noggin and ca-ALK-3, respectively. Finally, the infected cells were examined for cell proliferation, differentiation, and osteoclast-supporting activity.

For immunocytostaining, cells were fixed with $10 \%$ formalin for 10 minutes, treated with $0.1 \% \mathrm{H}_{2} \mathrm{O}_{2}$ for 30 minutes to remove endogenous peroxidase activity, and blocked with 5\% normal goat serum for 1 hour. They were then incubated with rat anti-human noggin antibody or anti-HA antibody (Santa Cruz Biotechnology Inc., Santa Cruz, California, USA) for 1 hour and subsequently with biotinylated second antibody (Vector Laboratories Inc., Burlingame, California, USA) for 30 minutes, followed by incubation with an $\mathrm{ABC}$ kit (Vector Laboratories Inc.). As a negative control, cells were incubated with rat IgG or mouse IgG instead of the primary antibody.

U-33 cells $\left(10^{4}\right.$ cells/well $)$ infected with virus containing empty vector, noggin, or $A L K-3$ were cultured in $\alpha$-MEM containing $10 \%$ FBS. Cell proliferation rates were not changed among the established cell lines. To compare osteoblast differentiation in the U-33 cells infected with empty vector and noggin vector, ALP activity and expression of Runx-2 (cbfa-1), bone sialoprotein, and osteocalcin (OG2) mRNA were measured using a colorimetric assay and real-time PCR. To examine osteoclast-supporting activity, osteoclast/macrophage progenitors described above were cultured with U-33 cells infected with empty vector or noggin vector in the presence of $10^{-8} \mathrm{M} 1,25(\mathrm{OH})_{2} \mathrm{D}_{3}$ for 1 week followed by counting of TRAP-stained cells. In addition, RANK-L expression in the U-33 vector or -noggin were measured by real-time PCR after 2 days in culture with $10^{-8} \mathrm{M} 1,25(\mathrm{OH})_{2} \mathrm{D}_{3}$.

We next examined whether ca-ALK-3 infection could prevent the effects of high-dose recombinant noggin on osteoblast differentiation. First, we treated mature OB-6 osteoblasts (16) with various concentrations of BMP- 2 or BMP- 4 in the absence or presence of a range of noggin or pegylated noggin (PEG-noggin) concentrations for 3 days and then measured ALP activity. Next, U-33-ca-ALK-3 cells were treated with recombinant PEG-noggin (0.1-100 ng/ml) for 3 days and ALP activity was measured. Human recombinant noggin and PEG-noggin were kindly provided by V. Economides (Regeneron Pharmaceuticals Inc.).

Phenotypic characterization of noggin-overexpressing mice. Noggin-overexpressing transgenic mice were generated in the National Institute of Child Health and Human Development Transgenic Mouse Development Facility, University of Alabama. The noggin transgene consisted of a 1.3-kb murine osteocalcin gene 2 (OG-2) promoter (23) (kindly provided by Gerard Karsenty, University of Texas, Houston, Texas, USA), murine noggin cDNA, and the human growth hormone (hGH) mini-gene (24). Before establishing the transgenic mice, 
we confirmed that the OG-2-noggin-hGH transgene $(4.3 \mathrm{~kb})$ was expressed in terminally differentiated, osteocalcin-expressing OB-6 cells (16), and not in undifferentiated mesenchymal 10T1/2 cells. Four lines carrying the OG-2-noggin transgene were provided as $\mathrm{C} 57 \mathrm{BL} / 6 \mathrm{~J} \times \mathrm{SJL} / \mathrm{J} \mathrm{F}_{2}$ hybrid founders. These were then bred for ten generations with $\mathrm{C} 57 \mathrm{BL} / 6 \mathrm{~J}$ mice to generate inbred colonies. The presence of the transgene was detected by PCR amplification and Southern blot analysis using tail genomic DNA. Radiologic, densitometric, histologic, and ex vivo experiments were carried out using 4- or 8-month-old littermates (10-15 animals per group). Bone mineral density was measured by a small animal densitometer (PIXImus; Lunar Corp., Madison, Wisconsin, USA). The precision of the densitometer is considered to be below $2.0 \%$.

Femur, tibia, and calvaria of WT and noggin-overexpressing mice were later dissected and fixed in 10\% formaldehyde, decalcified, and embedded in paraffin. Four-micrometer sections were stained with H\&E. To measure marrow space in frontal calvaria, $5-\mu \mathrm{m}$ serial sections from three different positions $500 \mu \mathrm{m}$ apart were analyzed. Marrow space and total bone area in each section were measured using a Nikon microscope with enhanced image analysis and expressed as a percentage of marrow space to the total bone area. Bone sections of femur, tibia, and spine were also prepared from animals injected intraperitoneally with $10 \mathrm{mg} / \mathrm{kg}$ calcein 7 days and 2 days before sacrifice. Bone formation was expressed as a ratio of the surface distance labeled with double calcein lines to the surface circumference (extent of bone formation), and as the interlabel distance (the distance between double calcein lines) (rate of bone formation).
CFU fibroblasts (CFU-F) and CFU osteoblasts (CFU$\mathrm{OB}$ ) in isolated bone marrow cells were quantitated following a 10-day or 28-day culture period under differentiating conditions (i.e., $1 \mathrm{mM}$ ascorbic acid-2-phosphate) $(15,20)$. CFU-F colonies were identified and counted after staining for ALP. Visualization of mineral deposition was achieved with von Kossa staining of CFU-OB colonies.

Statistical analyses. All in vitro and in vivo experiments were repeated at least three times. ANOVA was used to test for significant differences between two groups, and differences were considered significant at $P<0.05$.

\section{Results}

Noggin expression in noggin ${ }^{+/-} /$LacZ mouse bone. BMPs and noggin have a key role in embryonic skeletal development, hence the expression of both molecules is high during skeletal morphogenesis $(1,2)$. However, expression of $\mathrm{BMP} /$ noggin is very low in adult animals. We have therefore used a noggin ${ }^{+/-}$mouse with a LacZ transgene inserted at the site of noggin gene deletion to examine localization of noggin in bone tissues. The nog$\mathrm{gin}^{+/-} / \mathrm{LacZ}$ mice grew and developed normally. Figure 1 shows histological sections of spine and calvaria from 4-month-old adult mice stained for $\beta$-gal. Hypertrophic chondrocytes in spine were strongly positive for $\beta$-gal (Figure 1a), whereas those of WT mice were negative (Figure $1 \mathrm{~b}$ ). $\beta$-gal staining indicating noggin expression was also detected in osteoblasts in cortical and trabecular area of spine (Figure 1c). Periosteal fibroblasts and osteoblast precursors lining the outer and inner calvarial tables, in addition to the cells in cranial suture, were strongly $\beta$-gal-positive (Figure 1e). Polarized images of spine and calvaria (Figure 1, $\mathrm{d}$ and $\mathrm{f}$ ) showed the location of the respective cells in relation to collagen filaments.
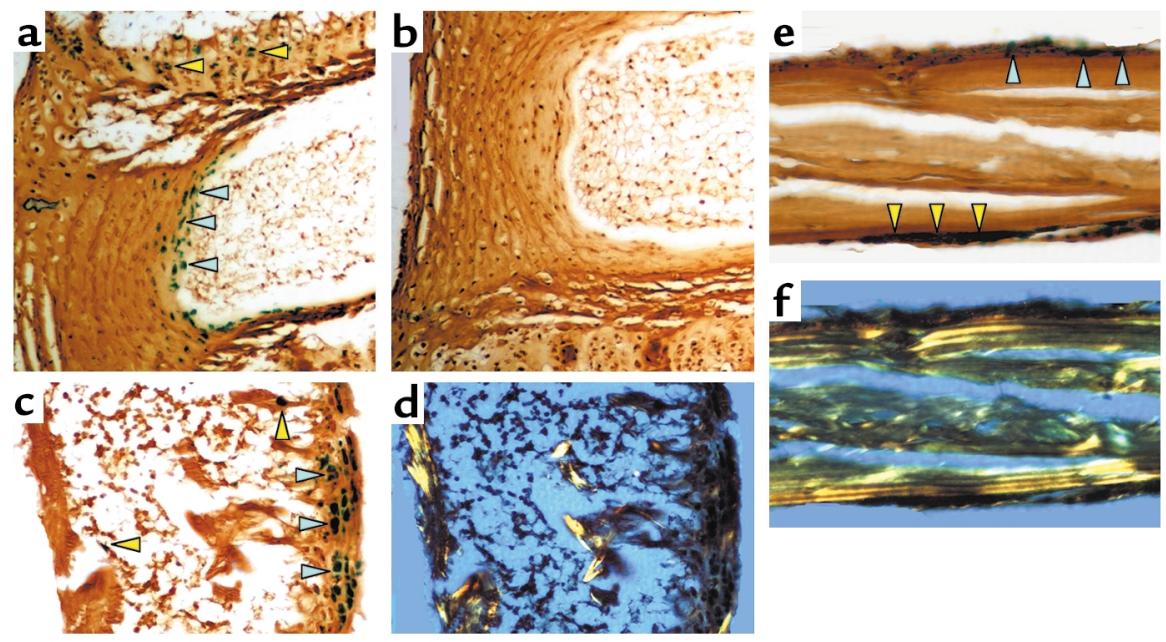

\section{Figure 1}

Noggin expression assessed by $\beta$-gal staining in adult bone tissues. Spine (a, b, $\mathbf{c}$, and $\mathbf{d})$ and calvaria (e and $\mathbf{f})$ from adult 4-month-old nog$\mathrm{gin}^{+/-}$mice (a, c-f) or noggin $\mathrm{it}^{++}$mice (as a negative control) (b) were stained for $\beta$-gal to investigate localization of cells expressing noggin. $\mathbf{d}$ and $\mathbf{f}$ represent polarized images corresponding to $\mathbf{c}$ and $\mathbf{e}$, respectively. In a, yellow and blue arrows indicate noggin-expressing hypertrophic chondrocytes and mesenchymal osteoblastic cells in the capsule around the vertebral disc, respectively. In c, blue and yellow arrows indicate noggin-expressing osteoblasts/osteocytes in cortical bone and osteoblasts in trabecular bone, respectively. In e, blue and yellow arrows indicate noggin-expressing osteoblasts of outer and inner calvaria, respectively. 

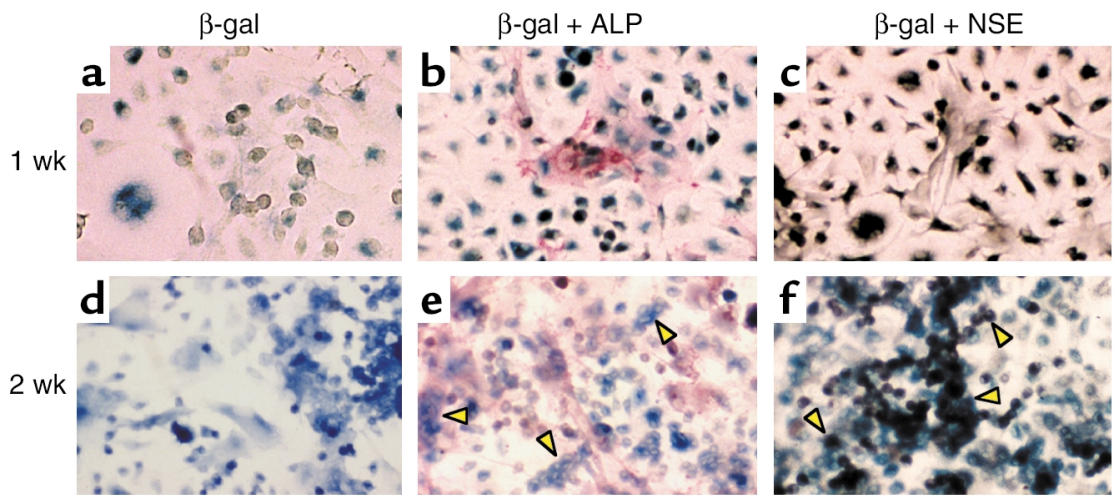

\section{Figure 2}

Noggin expression assessed by $\beta$-gal staining in bone marrow cell cultures. Bone marrow cells from adult noggin ${ }^{+/-}$mice were cultured for either 1 week $(\mathbf{a}-\mathbf{c})$ or 2 weeks $(\mathbf{d}-\mathbf{f})$ and stained for $\beta$-gal alone (blue, $\mathbf{a}$ and $\mathbf{d}$ ) or $\beta$-gal with either ALP (red, $\mathbf{b}$ and $\mathbf{e}$ ) or NSE (black in $\mathbf{c}$ and $\mathbf{f}$ ) to identify cell types in the culture. Yellow arrowheads indicate cells that costain with $\beta$-gal and ALP or $\beta$-gal and NSE.
In parallel experiments, bone marrow cells from adult noggin ${ }^{+/} /$LacZ mice were cultured for 1-2 weeks. The cells were costained for $\beta$-gal and either ALP or NSE (Figure 2). $\beta$-gal ${ }^{+}$cells appeared after about 1 week of culture, though LacZ staining was detectable after overnight incubation. $\mathrm{LacZ}^{+}$cells were small, spindle shaped, and costained only with NSE. After 2 weeks of culture, intense $\beta$-gal staining was visible in a large proportion of cells within 4 hours of incubation. These $\beta$-gal ${ }^{+}$cells were somewhat larger, round or spindle shaped, and likely represented a mix of different cell types. In fact, these cells costained for either ALP or NSE. This indicated that osteoblasts/stromal cells as well as macrophages (surprisingly) expressed noggin. To confirm directly that noggin was expressed in macrophages, we isolated macrophages from bone marrow and examined noggin mRNA and protein levels by real-time PCR and Western blotting, respectively. BMP-4 and noggin mRNA in macrophages was approximately $60 \%$ of that expressed in U-33 cells (Figure 3a). Western blotting, shown in Figure 3, b and $c$, not only confirmed the expression of the noggin protein in macrophages, but also indicated that noggin levels in macrophages could be upregulated by BMP-2. In parallel, we found that murine macrophage cell lines P388, J744, and RAW 264.7 expressed both BMP-4 and noggin mRNA and protein (data not shown). In contrast, osteoclasts formed in bone marrow cell cultures in the presence of M-CSF and RANK-L did not express noggin detectable by $\beta$-gal staining (data not shown).

\section{Figure 3}

Noggin expression in bone marrow-derived macrophages. Bone marrow-derived macrophages $(\mathrm{M} \phi)$ isolated from bone marrow cell cultures in the presence of M-CSF examined for noggin and BMP-4 expression by real-time PCR (a) and Western blot (b and $\mathbf{c}$ ). Expression levels in macrophages in a were described as a ratio to those in $\mathrm{U}-33$ preosteoblasts. In $\mathbf{b}$, noggin expression in macrophages and $\mathrm{U}-33$ cells were detected by Western blotting. In c, macrophages were cultured in the presence or absence of $300 \mathrm{ng} / \mathrm{ml} \mathrm{BMP-2}$ for 3 days, and noggin expression was analyzed by Western blotting. ${ }^{*} P<0.05$, significantly different from U-33 cells by ANOVA. Cont, control.
Regulation of noggin expression in cells isolated from nog$\mathrm{gin}^{+/-} /$LacZ mice. Neonatal calvarial osteoblasts were prepared from noggin $n^{+/} /$LacZ mice and were stained and counted as $\beta$-gal ${ }^{+}$cells. The $\beta$-gal ${ }^{+}$osteoblast count increased from $2 \%$ to $8 \%$ of total cells during the 6 -day culture under basal conditions (Figure 4a). The application of BMP-2 $(300 \mathrm{ng} / \mathrm{ml})$ resulted in a dramatic enhancement of the $\beta$-gal ${ }^{+}$osteoblast count. That these cells were mature rather than immature osteoblasts was evident from a parallel enhancement in the expression of late osteoblastic markers, including bone sialoprotein and osteocalcin (data not shown).

The detailed time course shown in Figure $4 \mathrm{~b}$ indicates that a significant $(P<0.05)$ effect of BMP-2 on noggin expression is evident as early as 1 day in culture, and that this increases exponentially with time up to 3 days (Figure $4 \mathrm{~b}$ ). In contrast, the related growth factor TGF- $\beta$ had no effect on the $\beta$-gal ${ }^{+}$osteoblast count in these cultures over the 3 -day time period. We further examined the effect of other hormones and growth factors, including BMP-4 and -6, IGF-1, parathyroid hormone, $1,25(\mathrm{OH})_{2} \mathrm{D}_{3}$, and noggin itself, on the appearance of $\beta$-gal ${ }^{+}$activity (Figure $4 c$ ). It is notable that only BMP- $2,-4$, and BMP- 6 had a profound effect on $\beta$-gal (noggin) expression that correlated strongly, at least at this 72-hour timepoint, with ALP activity.

Effects of noggin overexpression on osteoblast maturation. To study the effect of noggin on osteoblast differentiation, we overexpressed noggin in U-33 preosteoblasts using a retroviral vector, pLXSN. Figure 5, a and b, shows that U-33 cells have high noggin expression evi- 

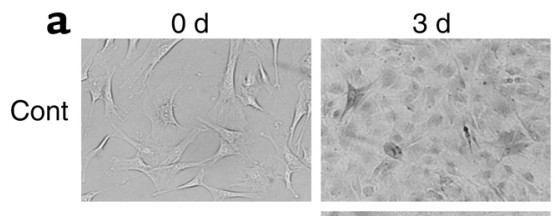

BMP-2

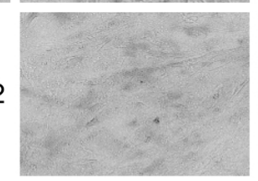

b

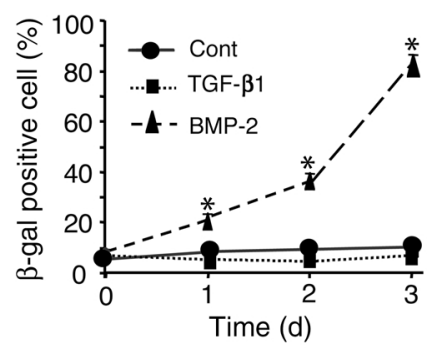

$6 \mathrm{~d}$

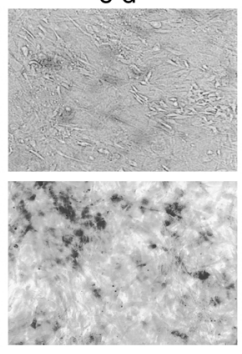

C

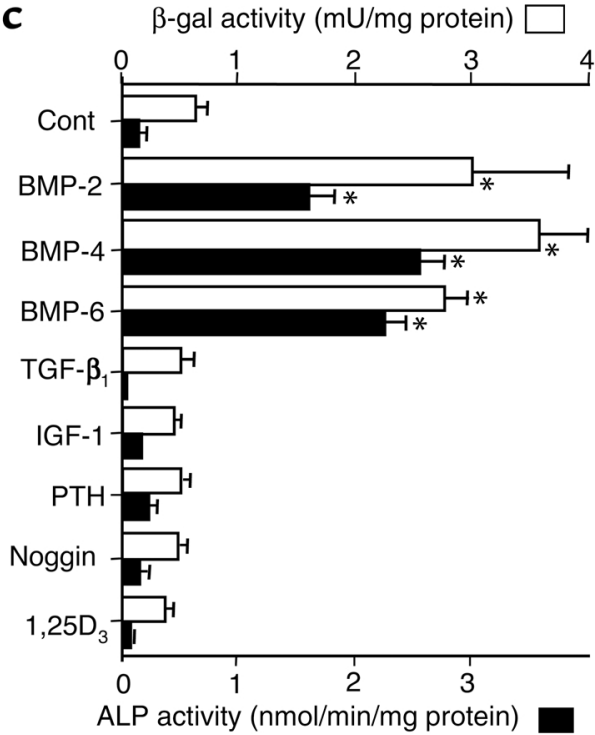

Figure 4

Time course of noggin expression and cell differentiation in calvarial osteoblasts. Calvarial osteoblasts from neonatal noggin ${ }^{+/-}$mice were cultured for up to 6 days in the presence of various osteotropic factors. (a) Isolated osteoblasts were cultured for 3 and 6 days in the presence or absence of BMP-2. (b) Time course of $\beta$-gal-positive cell numbers in the absence or presence of BMP- 2 and TGF- $\beta 1$. (c) $\beta$-gal and ALP activ-

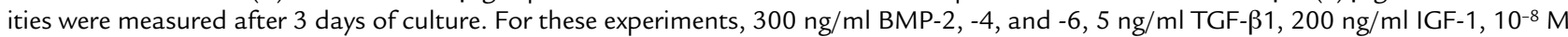
1-34 human parathyroid hormone (PTH), $300 \mathrm{ng} / \mathrm{ml}$ noggin, and $10^{-8} \mathrm{M} 1,25(\mathrm{OH})_{2} \mathrm{D}_{3}\left(1,25 \mathrm{D}_{3}\right)$ were applied. ${ }^{*} P<0.05$, significantly different from 0 timepoint $(\mathbf{b})$ or control group $(\mathbf{c})$.

dent upon Western blot and immunocytostaining. Noggin-overexpressing cells showed a marked (70-80\%) reduction in the expression of Runx-2, as well as the osteoblast differentiation markers bone sialoprotein and osteocalcin (Figure 5c). We also examined the osteoclast-supporting activity of the noggin-overex- pressing U-33 cells using a coculture system in which bone marrow osteoclast/macrophage progenitors were incubated with $1,25(\mathrm{OH})_{2} \mathrm{D}_{3}$. Figure $5 \mathrm{~d}$ shows a dramatic reduction in TRAP-positive osteoclast formation in cocultures that used noggin-overexpressing U-33 cells compared with those using vector-infected control a
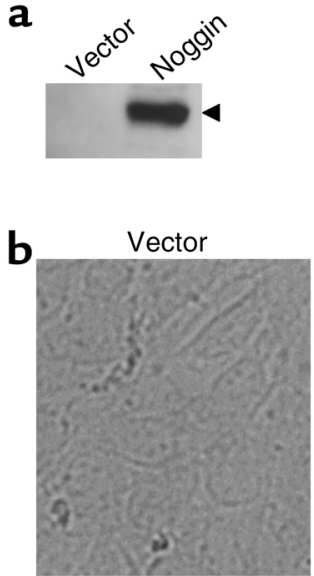

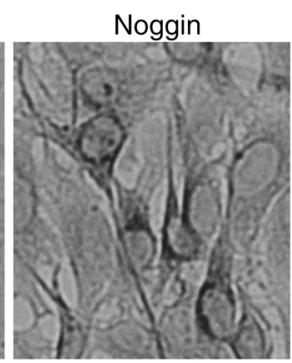

C
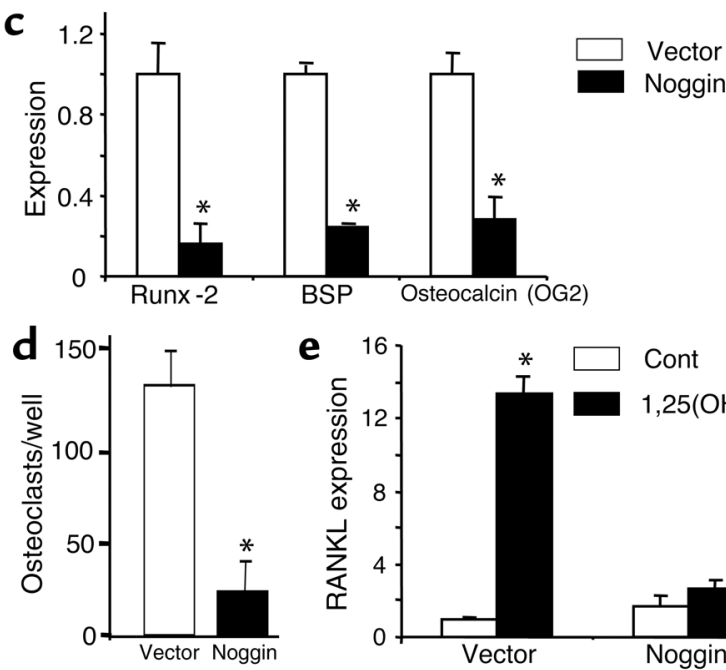

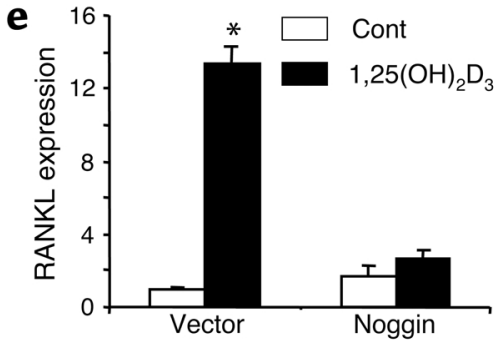

Figure 5

Effects of overexpression of noggin in U-33 cells. Overexpression of noggin in U-33 cells generated by a virus infection system (see Methods) was detected by Western blotting (a) and immunocytostaining (b) using an anti-noggin monoclonal antibody. Cells infected with empty viral vector (Vector) were used as control. Arrow indicates $30 \mathrm{kDa}$ protein under reducing conditions (a). Expression of Runx-2, bone sialoprotein (BSP), and osteocalcin (c), osteoclast-supporting activity (d), and expression of RANK-L in the presence of $10^{-8} \mathrm{M} 1,25(\mathrm{OH})_{2} \mathrm{D}_{3}(\mathbf{e})$ were compared in the control and noggin-infected U-33 cells. The expression levels of Runx-2, BSP, osteocalcin, and RANK-L were measured by real-time PCR and expressed as a ratio to untreated control (Vector) cells. TRAP-positive osteoclast number and RANK-L expression are expressed as mean \pm SEM. ${ }^{*} P<0.05$, significantly different from untreated control cells. 

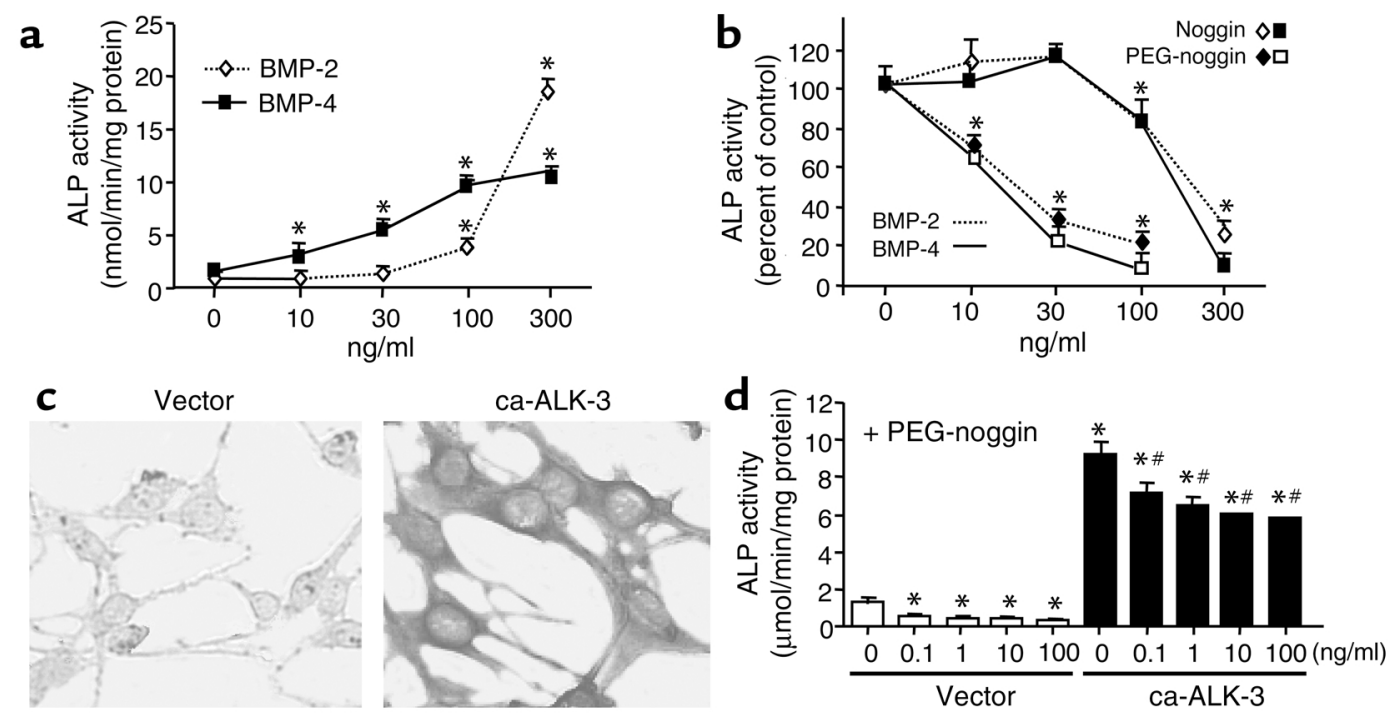

\section{Figure 6}

Effects of BMP and ca-ALK-3 overexpression on osteoblast differentiation. (a) Concentration-dependent stimulation of ALP activity in mature OB- 6 osteoblasts by BMP-2 and BMP-4, expressed as absolute values. (b) Concentration-dependent inhibition of ALP activity by noggin or PEG-noggin in the presence of a fixed dose of BMP-2 or BMP-4 (100 ng/ml), expressed as a percentage of the control value. (c) Anti-HAimmunostained U-33 cells documenting constitutively active BMP type 1A receptor (ca-ALK-3) expression; the left panel (Vector) is a negative control. (d) Effect of increasing concentrations $(0-100 \mathrm{ng} / \mathrm{ml}$ for 3 days) of PEG-noggin in vector or ca-ALK-3-overexpressing U-33 cells as assessed by ALP activity (given as mean \pm SEM), expressed as absolute values. ${ }^{*} P<0.05$, significantly different from untreated control (Vector) cells; ${ }^{P} P<0.05$, significantly different from ca-ALK-3 at 0 concentration, by ANOVA.

cells. This data was consistent with the reduction in RANK-L expression in noggin-overexpressing cells in response to $1,25(\mathrm{OH})_{2} \mathrm{D}_{3}$ (Figure 5e).

Osteoblast differentiation is determined by the balance between BMP and noggin expression. We therefore asked several questions: (a) whether BMP-2 and -4 actions were inhibited by noggin (b) whether PEG-noggin was more potent than noggin, and (c) whether caAlk-3 stimulated osteoblast differentiation despite the presence of noggin. We found that BMP-2 and BMP-4 were equivalent in enhancing ALP activity in mature OB-6 osteoblasts (Figure 6a). PEG-noggin was approximately tenfold more potent than noggin in inhibiting the effects of BMP-2 and BMP-4 (Figure 6b). Finally, infection of cells with the ca-ALK-3 construct (Figure 6c) resulted in a dramatic increase in ALP activity (Figure $6 \mathrm{~d}$ ). Notably, the increased ALP activity of these cells remained high (between $60 \%$ and $80 \%$ of control values), even in the presence of a high concentration of PEG-noggin $(100 \mathrm{ng} / \mathrm{ml})$. The initial small inhibition of ALP activity at concentrations of $0-0.1 \mathrm{ng} / \mathrm{ml}$ PEGnoggin may be due to the inhibition of the endogenously produced BMPs by PEG-noggin.

In vivo and in vitro characterization of the noggin-overexpressing mouse. The noggin transgene consisted of a 1.3$\mathrm{kb}$ murine osteocalcin gene $2(O G-2)$ promoter, murine noggin $\mathrm{cDNA}$, and the hGH mini-gene. The value of using the murine $O G-2$ promoter has been to specifically localize gene expression to mature bone-forming, osteocalcin-positive osteoblasts. Four lines carrying the OG-2-noggin transgene were provided as $\mathrm{C} 57 \mathrm{BL} / 6 \mathrm{~J} \times$ $\mathrm{SJL} / \mathrm{J} \mathrm{F}_{2}$ hybrid founders. These were then bred for ten generations with C57BL/6J mice to generate inbred colonies. Line 1 and line 5 showed between five and seven copies of the transgene on PCR and Southern blotting of tail DNA. More detailed analysis was performed on line 5. (Figure 7a depicts RT-PCR and Western blots demonstrating high - greater than tenfold transgene expression in bone, but not in liver or kidney.)

Phenotypic characterization of 4-month-old transgenic littermates revealed normal bone growth, radiological appearance, and bone mineral density at the femur, tibia, and lumbar spine (Figure 7, b and c). Figure $7 \mathrm{~d}$ shows the disorganized endochondral bone in the noggin transgenic mice. We next isolated bone marrow cells from noggin transgenic mice and their WT littermates and examined ALP-positive CFU-F colonies after 10 days in culture and mineralizing $\mathrm{CFU}-\mathrm{OB}$ colonies after 28 days in culture. We found no defect in CFU-F formation but found reduced CFU-OB (Figure 7e). The dramatic suppression of mineralizing CFU-OB colonies was consistent with the appearance of noggin with osteocalcin during late osteoblastic differentiation. Figure $7 \mathrm{f}$ shows the formation of TRAP-positive osteoclasts from hematopoietic precursors in the presence of M-CSF and RANK-L. There was no difference in osteoclast formation between bone marrow cells derived from noggin-overexpressing mice and those from WT littermates.

Marked osteopenia and significant reductions in bone mineral density from 8-month-old noggin transgenic mice can be seen in Figure 8, a and b. In contrast to the 4-month-old animals, the older mice showed a loss of trabecular structure (Figure 8c). Furthermore, 
numbers of CFU-OB, CFU-F, and osteoclasts were significantly decreased (by about 50\%) (Figure 8, $\mathrm{d}$ and e). Thus, it is clear that overexpression of noggin with aging results in dramatic reductions in both osteoblast and osteoclast differentiation in vivo. In addition, bone formation parameters such as calcein-labeled surface and interlabel distance were significantly reduced (Figure 8, fand g). Interestingly, marrow space was significantly increased, even in the calvarial tissue of transgenic mice (Figure 8h).

Noggin overexpression during normal and accelerated aging. Reduced osteoblast differentiation is reminiscent of the low-turnover bone loss seen in aging humans $(25,26)$ and in aging or accelerated-aging mice such as the SAM-P6 mouse (27). To elucidate the effects of noggin, BMP-2, and BMP-4 as a function of aging, we examined their expression using normally aged 20-month-old C57BL/6J mice and 4-month-old SAM-P6 mice. The Western blots in Figure 9, a-c, show significantly higher levels of noggin expression in the calvaria and long bones of normally aged and SAM-P6 mice than in 4-month-old C57BL/6J and SAM-R1 mice, respectively. In parallel, noggin mRNA levels in bone marrow cells isolated from SAM-P6 mice, measured by real-time PCR, were also significantly elevated (two- to fourfold) (Figure 9d). In contrast, BMP-4 mRNA measurements revealed no significant differences between the respective mice. Similar results were obtained with bone marrow cell cultures isolated from aged 20-month-old C57BL/6J mice compared with 4-month-old adult mice (Figure 9e). Together, the results suggested (a) that noggin expression could be regulated independently of BMP- 2 and -4 , and (b) that the selective enhancement of noggin expression during aging could potentially inhibit BMP-induced osteoblast differentiation and bone formation.

\section{Discussion}

We demonstrate that noggin, expressed mainly in the osteoblasts, chondrocytes, and cells of mesenchymal origin, is an important regulator of osteoblast differentiation and bone formation in the adult skeleton. Noggin is known to play a key role in skeletal morphogenesis; however, it has not been possible to study its role in the adult skeleton because of postnatal lethality and because heterozygotic mice fail to exhibit a phenotype, possibly due to the redundancy in bone formation. We have previously shown that osteoblast differentiation in vitro is inhibited and stimulated, respectively, by recombinant noggin and an anti-noggin antibody (15). Additionally, we found that noggin inhibits osteoclastogenesis by reducing RANK-L expression in osteoblasts (15).

We show that noggin is expressed in several cell types in the adult skeleton. As its expression is generally low, it has previously been impossible to use traditional immunodetection and in situ hybridization to examine the cellular localization of noggin in the normal skeleton. However, noggin has been detected immunocytochemically at fracture sites and also where BMP-induced formation is high $(28,29)$. We thus adopted a highly sensitive and specific method of visualizing $\beta$-gal expression in a heterozygotic mouse in which LacZ was inserted at the site of noggin gene deletion (14). As expected, we noted noggin expression in mesenchymal cells, osteoblasts, and chondrocytes. Additionally, as others have shown (9), noggin was expressed in cranial sutures (data not shown). Mature osteoclasts did not express noggin. Surprisingly, however, we found not only by $\beta$-gal staining, but also directly by real-time PCR and Western blotting, that macrophages expressed noggin to a considerable extent. The relevance of noggin expres-
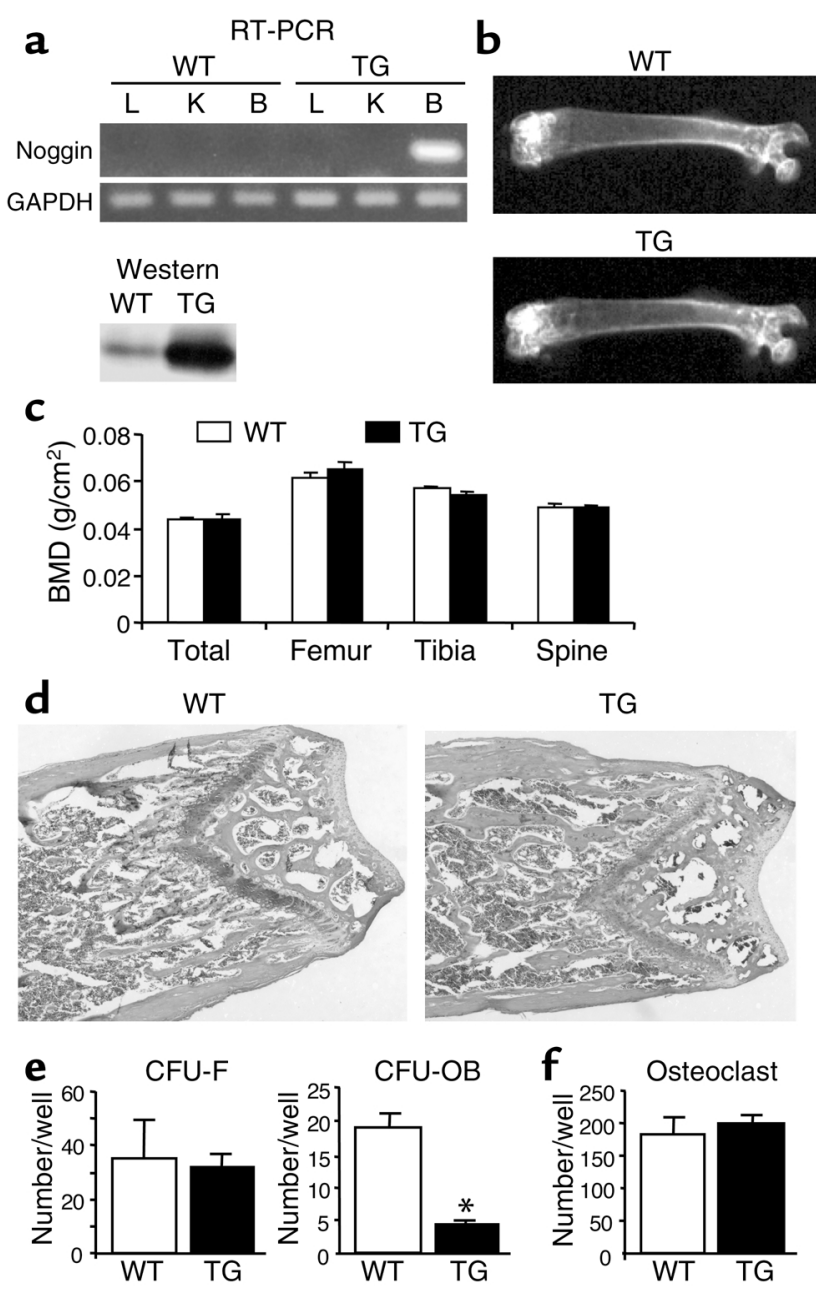

$\mathrm{TG}$

Figure 7

Phenotype of a 4-month-old noggin-overexpressing mouse. Expression of noggin mRNA detected by RT-PCR in liver (L), kidney (K), and bone (B), and by Western blotting in bone (a) in WT and noggin transgenic (TG) littermates; GAPDH was used as a control. (b) Radiology of the femur; (c) bone mineral density of the femur, tibia, spine (L4-L6), and total body; and (d) H\&E staining of femur were performed to show differences between WT and noggin transgenic mice. Comparison of bone marrow cell cultures isolated from WT and noggin transgenic mice to evaluate ALP-positive CFU-F, CFU-OB (e), and TRAP-positive osteoclast formation $(\mathbf{f})$. Results are expressed as mean \pm SEM. ${ }^{*} P<0.05$, significantly different from WT mice by ANOVA. 


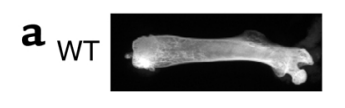

b

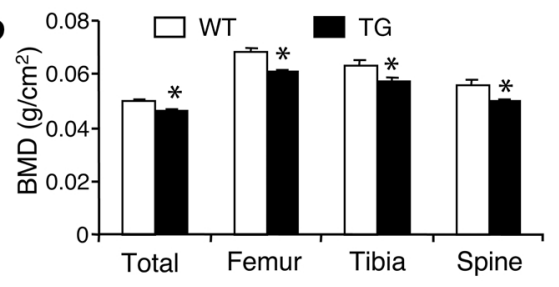

c

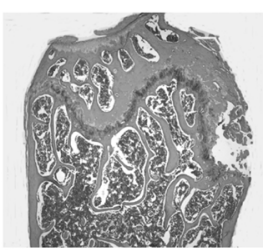

WT
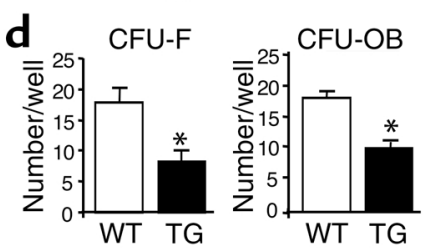
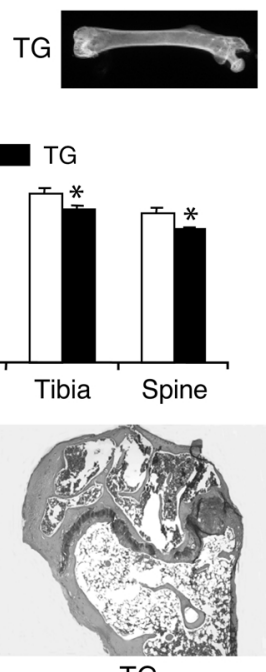

TG

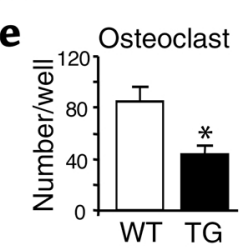

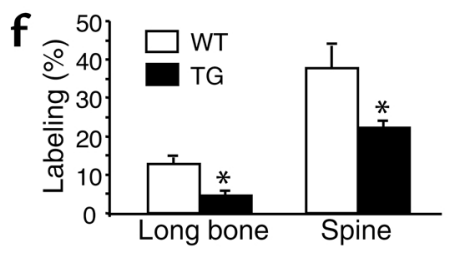
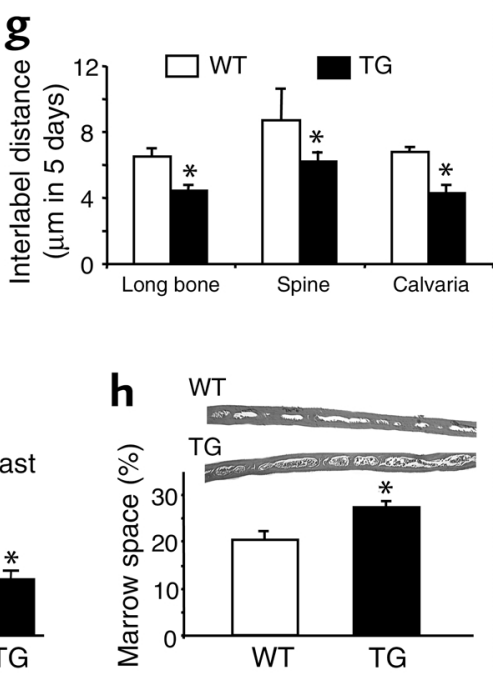

\section{Figure 8}

Phenotype of an 8-month-old nogginoverexpressing mouse. (a) Radiology of the femur; (b) bone mineral density of the femur, tibia, spine, and total body; and (c) H\&E staining of femur were performed to show differences in WT and noggin transgenic mice. (d) Comparison of bone marrow cell cultures isolated from WT and noggin TG mice to evaluate ALP-positive CFU-F, CFU-OB, and (e) TRAP-positive osteoclast formation. (f) The percentage of doublylabeled bone surface to total bone surface and $(\mathbf{g})$ the rate of bone formation as determined by the distance between calcein labeling lines in long bone (femur and tibia), spine, and calvaria from 8-month-old WT or noggin-overexpressing TG mice. Calvarial sections stained with $\mathrm{H} \& \mathrm{E}$ were analyzed to determine marrow space as a percentage of total bone area (h). Results are expressed as mean \pm SEM. Statistics by ANOVA between WT and noggin TG mice. ${ }^{*} P<0.05$ sion in the macrophage is yet to be established, but it is possible that it may modulate the known effects of BMP on osteoclast formation (30).

We next examined the regulation of noggin expression in $\beta$-gal-positive osteoblasts in response to BMP-2, BMP-4, and BMP-6, as well as other osteotropic hormones, including a related member, TGF- $\beta$. We found that $\beta$-gal/noggin levels were significantly enhanced by the BMPs, but not by TGF- $\beta$. Similar results showing that noggin is regulated by BMPs have been reported (31). Likewise, in differentiating bone cell cultures and during fracture healing, the expression of noggin follows that of BMP-4 and BMP-2, providing indirect evidence for the regulation of noggin by BMP. The molecular mechanism is unclear, although our cloning and sequencing of the murine noggin promoter has revealed several potential BMP-responsive sites (unpublished observations), including a Smad-binding element (SBE), a Runx-2 binding site (OG-2), and a Hox-c8 site (32-35). That BMP regulates the expression of its inhibitor, noggin, makes plausible the existence of a feedback regulatory mechanism. BMP stimulates noggin

\section{Figure 9}

Noggin expression in SAM-P6 mice and aged mice. Cell lysates (100 $\mu$ g protein) prepared from long bone (a) and calvaria (b and $\mathbf{c}$ ) of SAM-R1 (R1) and SAM-P6 (P6) mice (a and b) or from young (4-month-old) and old (20-month-old) mice (c) were subjected to Western blot to detect noggin protein expression. Band intensities of noggin expression in the top panels, which represent samples from individual animals, were quantified for $\beta$-actin expression and expressed in the graphs as a ratio of noggin to $\beta$-actin. (d and $\mathbf{e}$ ) Noggin and BMP-4 expression in bone marrow cell cultures of young and old mice (C57BL/6J) or SAM-R1 (R1) and SAM-P6 (P6) mice were analyzed by real-time RT-PCR after culturing with $1 \mathrm{mM}$ ascorbic acid-2-phosphate for 4 weeks. Gene expression is indicated as a ratio to the young or $\mathrm{R} 1$ control mice. ${ }^{*} P<0.05$, significantly different from SAM-R1 or young control mice.
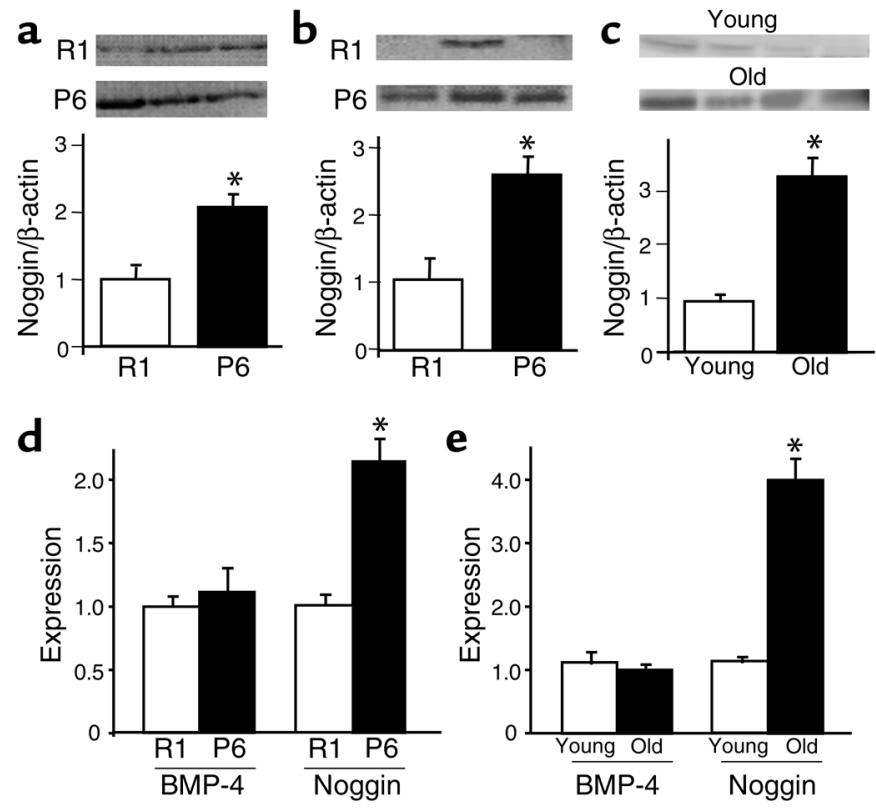
gene expression by eliciting a signaling cascade involving Smad-1, Smad-5, and Smad-8 (36) as well as the transcription factor osterix (37). Secreted noggin will then inhibit further BMP action, as we have shown in vitro using noggin-overexpressing osteoblastic cells.

It may be deduced therefore that the balance between BMP and noggin is critical in preventing overtly high levels of bone formation, a situation akin to that of osteoprotegerin, which as a decoy receptor for RANK-L prevents excessive osteoclastogenesis and bone resorption (38). Recent studies using male mice showed that aged animals exhibited increased RANK-L expression but decreased osteoprotegerin expression in bone and bone marrow-derived osteoblasts (39). To this end, we find that normal age-related osteopenia is associated with a dramatic increase in noggin expression in bone, with no change in BMP-4 levels. Similarly, the accelerated osteopenia seen at 4 months in the SAM-P6 mouse is also associated with high bone levels of noggin and normal BMP-4 levels. These striking parallels in two separate aging models suggest strongly that a relative elevation of noggin contributes, at least in part, to lowturnover involutional osteopenia. The reason for BMPindependent enhancements in noggin expression during aging remains unclear. It could result from either an increased number of mature noggin-producing osteoblasts or the induction of noggin at an earlier stage of osteoblast differentiation. Our studies with nogginoverexpressing mice show that osteopenia develops as a function of aging even with overexpression of noggin selectively in osteoblasts. Similarly, severe defects in bone formation have recently been reported using a rat osteocalcin promoter (40). These authors found more severe bone defects; results differing from ours likely because of the promoters used (rat versus mouse).

The lack of osteopenia at 4 months is not unexpected as noggin is overexpressed only in mature mineralizing osteoblasts; these represent a minor and localized population of all osteoblastic cells in situ. Furthermore, the inhibitory effect of noggin may be overcome by the enhancement of other BMPs that may signal through the same receptor, ALK-3. Nonetheless, despite the lack of an overall phenotype, when osteoblasts from the transgenic mouse are allowed to differentiate in culture, the formation of mineralizing CFU-OB colonies is significantly decreased, even at 4 months. At 8 months, however, severe radiologic and densitometric osteopenia results, with evidence of trabecular bone loss and a pronounced bone formation defect. The numbers of both osteoblast progenitors, CFU-F and CFU-OB, are decreased by approximately $50 \%$. Taken together, our results are consistent with an important function of noggin in osteoblast differentiation and bone formation that occurs as a function of aging.

What, therefore, is the physiological role of noggin in skeletal remodeling? We know that noggin is involved in skeletal morphogenesis. Newborn noggin knockout mice show evidence of joint deformity (14). Similarly, in humans, a non-dominant noggin gene mutation results in joint deformities (41). Here, we show for the first time, to our knowledge, that noggin is expressed in cells of the adult mouse skeleton. We also demonstrate using noggin-overexpressing cells and a transgenic mouse that noggin inhibits osteoblast differentiation both in vitro and ex vivo. Importantly, we have previously found that there is a temporal dissociation between the appearance of noggin and BMP: in all instances examined so far, such as during fracture healing and in differentiating bone marrow cell cultures, noggin expression follows that of BMP. It makes sense, therefore (and we have shown this to be the case ex vivo through $\beta$-gal measurements in noggin ${ }^{+-}$mice), that BMPs directly regulate noggin expression, likely as part of a feedback mechanism to prevent excessive BMP action.

Thus, noggin is involved in normal adult bone remodeling, particularly during the phases of bone formation. Likewise, there is direct evidence that Tob, a downstream Smad inhibitor, when knocked out specifically in osteoblasts, results in enhanced bone formation in adult mice (42). It is also plausible that a chronic imbalance between the expression of noggin and BMPs contributes to the osteopenia seen during biological aging. Should this be the case, the next question is whether recombinant BMP can be used to reverse age-related osteopenia. In fact, recombinant BMP injections into aged mice have been shown to increase bone formation modestly with a higher dose requirement in aged mice than in young animals (18), likely because of noggin overexpression.

\section{Acknowledgments}

This work was supported by Veterans Administration Merit Review Grants to E. Abe and M. Zaidi and by NIH grant AG-14197-08 to M. Zaidi. E. Abe wishes to acknowledge colleagues at the University of Arkansas for Medical Sciences for advice and help, notably Charles A. O'Brien for helpful discussion and suggestions in producing the noggin-transgenic mice, Robert S. Weinstein for sectioning of tibia of noggin ${ }^{+/-}$ mice, and Stavros C. Manolagas for financial support and helpful critique.

1. Wozney, J.M. 1993. Bone morphogenetic proteins and their gene expression. In Cellular and molecular biology of bone. M. Noda, editor. Academic Press Inc. San Diego, California, USA. 131-167.

2. Rosen, V., Cox, K., and Hattersley, G. 1996. Bone morphogenetic proteins. In Principles of bone biology. J.P. Bilezikin, L.G. Raisz, and G.A. Rodan, editors. Academic Press Inc. San Diego, California, USA. 661-671.

3. Winnier, G., Blessing, M., Labosky, P.A., and Hogan, B.L.M. 1995. Bone morphogenetic protein- 4 is required for mesoderm formation and patterning in the mouse. Genes Dev. 9:2105-2116.

4. Mishina, Y., Suzuki, A., Ueno, N., and Behringer, R.R. 1995. BMPR encodes a type I bone morphogenetic protein receptor that is essential for gastrulation during mouse embryogenesis. Genes Dev. 9:3027-3037.

5. Botchkarev, V.A., et al. 1999. Noggin is a mesenchymally derived stimulator of hair-follicle induction. Nat. Cell Biol. 1:158-164.

6. Valenzuela, D.M., et al. 1995. Identification of mammalian noggin and its expression in the adult nervous system. J. Neurosci. 15:6077-6084.

7. Jamali, M., Karamboulas, C., Rogerson, P.J., and Skerjanc, I.S. 2001. BMP signaling regulates $\mathrm{Nkx2}-5$ activity during cardiomyogenesis. FEBS Lett. 509:126-130.

8. Hager-Theodorides, A.L., et al. 2002. Bone morphogenetic protein $2 / 4$ signaling regulates early thymocyte differentiation. J. Immunol. 169:5496-5504. 
9. Warren, S.M., Brunet, L.J., Harland, R.M., Economides, A.N., and Longaker, M.T. 2003. The BMP antagonist noggin regulates cranial suture fusion. Nature. 10:625-629.

10. Smith, W.C., and Harland, R.M. 1992. Expression cloning of noggin, a new dorsalizing factor localized to the Spemann organizer in Xenopus embryos. Cell. 70:829-840.

11. Canalis, E., Economides, A.N., and Gazzero, E. 2003. Bone morphogenetic proteins, their antagonists, and the skeleton. Endocr. Rev. 24:218-235.

12. Zimmerman, L.B., De Jesus-Escobar, J.M., and Harland, R.M. 1996. The Spemann organizer signal, noggin, binds and inactivates bone morphogenetic protein 4. Cell. 86:599-606.

13. Re'em-Kalma, Y., Lamb, T., and Frank, D. 1995. Competition between noggin and bone morphogenetic protein 4 activity may regulate dorsalization during Xenopus development. Proc. Natl. Acad. Sci. U. S. A. 92:12141-12145.

14. Brunet, L.J., McMahon, J.A., McMahon, A.P., and Harland, R.M. 1998. Noggin, cartilage morphogenesis, and joint formation in the mammalian skeleton. Science. 280:1455-1457.

15. Abe, E., et al. 2000. Requirement of BMPs-2/4 for postnatal osteoblast and osteoclast formation: antagonism by noggin. J. Bone Miner. Res. 15:663-673.

16. Lecka-Czernik, B., et al. 1999. Inhibition of Osf2/cbfa1 expression and terminal osteoblast differentiation by PPAR-gamma 2. J. Cell. Biochem. 74:357-371.

17. Kimble, R.B., et al. 1998. Noggin inhibits bone formation in a model of heterotopic ossification. J. Bone Miner. Res. 12:s244. (Abstr.)

18. Fleet, J.C., Cashman, K., Cox, K., and Rosen, V. 1996. The effects of aging on the bone inductive activity of recombinant human bone morphogenetic protein-2. Endocrinology. 137:4605-4610.

19. Yamate, T., et al. 1997. Osteopontin expression by osteoclast and osteoblast progenitors in the murine bone marrow: demonstration of its requirement for osteoclastogenesis and its increase following ovariectomy. Endocrinology. 138:3047-3055.

20. Di Gregorio, G.B., et al. 2001. Attenuation of the self-renewal of transitamplifying osteoblast progenitors in the murine bone marrow by $17 \beta$ estradiol. J. Clin. Invest. 107:803-812.

21. Abu-Amer, F.Y., et al. 1998. Tumor necrosis factor- $\alpha$ activation of nuclear transcription factor- $\kappa \mathrm{B}$ in marrow macrophages is mediated by $\mathrm{c}-\mathrm{Src}$ tyrosine phosphorylation of IкB $\alpha$. J. Biol. Chem. 273:29417-29423.

22. Wan, M., Shi, X., Feng, X., and Cao, X. 2001. Transcriptional mechanisms of bone morphogenetic protein-induced osteoprotegerin gene expression. J. Biol. Chem. 276:10119-10125.

23. Schinke, T., and Karsenty, G. 1999. Characterization of Osf1, an osteoblast-specific transcription factor binding to a critical cis-acting element in the mouse Osteocalcin promoters. J. Biol. Chem. 274:30182-30189.

24. Chaffin, K.E., et al. 1990. Dissection of thymocyte signaling pathways by in vivo expression of pertussis toxin ADP-ribosyltransferase. EMBOJ. 9:3821-3829.

25. Parfitt, A.M., et al. 1983. Relationships between surface, volume, and thickness of iliac trabecular bone in aging and in osteoporosis. Implica- tions for the microanatomic and cellular mechanism of bone loss. J. Clin Invest. 72:1396-1409.

26. Eriksen, E.F., et al. 1990. Cancellous bone remodeling in type I (postmenopausal) osteoporosis: quantitative assessment of rates of formation, resorption, and bone loss at tissue and cellular levels. J. Bone Miner Res. 5:311-319.

27. Jilka, R.L., Weinstein, R.S., Takahashi, K., Parfitt, A.M., and Manolagas, S.C. 1996. Linkage of decreased bone mass with impaired osteoblastogenesis in a murine model of accelerated senescence. J. Clin. Invest. 97:1732-1740

28. Nakase, T., et al. 1997. Transient and localized expression of bone morphogenetic protein 4 messenger RNA during fracture healing. J. Bone Miner. Res. 9:651-659.

29. Yoshimura, Y., et al. 2001. Colocalization of noggin and bone morphogenetic protein-4 during fracture healing. J. Bone Miner. Res. 16:876-884.

30. Itoh, K., et al. 2001. Bone morphogenetic protein 2 stimulates osteoclast differentiation and survival supported by receptor activator of nuclear factor-kappaB ligand. Endocrinology. 142:3656-3662.

31. Gazzerro, E., Gangji, V., and Canalis, E. 1998. Bone morphogenetic proteins induce the expression of noggin, which limits their activity in cultured rat osteoblasts. J. Clin. Invest. 102:2106-2114.

32. Jonk, L.J., Itoh, S., Heldin, C.H., ten Dijke, P., and Kruijer, W. 1998. Identification and functional characterization of a Smad binding element (SBE) in the JunB promoter that acts as a transforming growth factorbeta, activin, and bone morphogenetic protein-inducible enhancer. J. Biol. Chem. 273:21145-21152.

33. Frendo, J.L, et al. 1998. Functional hierarchy between two OSE2 elements in the control of osteocalcin gene expression in vivo. J. Biol. Chem. 273:30509-30516.

34. Shi, X., Yang, X., Chen, D., Chang, Z., and Cao, X. 1999. Smad1 interacts with homeobox DNA-binding proteins in bone morphogenetic protein signaling. J. Biol. Chem. 274:13711-13717.

35. Hullinger, T.G., Pan, Q., Viswanathan, H.L., and Somerman, M.J. 2001. TGFbeta and BMP-2 activation of the OPN promoter: roles of smadand hox-binding elements. Exp. Cell Res. 262:69-74.

36. Ishida, W., et al. 2000. Smad6 is a Smad1/5-induced smad inhibitor. Characterization of bone morphogenetic protein-responsive element in the mouse Smad6 promoter. J. Biol. Chem. 275:6075-6078.

37. Nakashima, K., et al. 2002. The novel zinc finger-containing transcription factor osterix is required for osteoblast differentiation and bone formation. Cell. 108:17-29.

38. Simonet, W.S., et al. 1997. Osteoprotegerin: a novel secreted protein involved in the regulation of bone density. Cell. 89:309-319.

39. Cao, J., Venton, L., Sakata, T., and Halloran, B.P. 2003. Expression of RANKL and OPG correlates with age-related bone loss in male C57BL/6 mice. J. Bone Miner. Res. 18:270-277.

40. Devlin, R.D., et al. 2003. Skeletal overexpression of noggin results in osteopenia and reduced bone formation. Endocrinology. 144:1972-1978.

41. Gong, Y., et al. 1999. Heterozygous mutations in the gene encoding noggin affect human joint morphogenesis. Nat. Genet. 21:302-304.

42. Yoshida, Y., et al. 2000. Negative regulation of BMP/Smad signaling by Tob in osteoblasts. Cell. 103:1085-1097. 